\title{
Proteomics focusing on immune markers in psoriatic arthritis
}

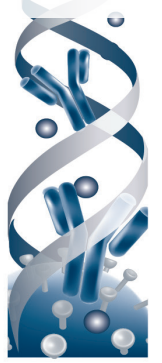

The immune-pathogenesis of psoriatic arthritis represents a subject of intense research, as a still unknown factor can trigger the chronic inflammation that, upon a defective immune terrain, generates this auto-immune/auto-inflammatory condition. The pathogenesis complexity of psoriatic arthritis resides in the psoriatic synovitis milieu, where intricate immune relations are emerging during disease development. Innate immune response generates inflammatory cytokines driving effectors functions for immune and non-immune cells that sustain the chronical character of the synovitis. Herein, we review the updated information regarding biomarkers/ immune markers that sustain the heterogeneity and complexity of psoriatic arthritis pathogenesis, this complexity leading to multifaceted methodological approaches for disease investigation. New immune proteomic or genomic biomarkers can enlarge and identify new therapeutic targets.

Keywords: diagnosis $\bullet$ immune markers $\bullet$ immune therapy $\bullet$ proteomics $\bullet$ psoriatic arthritis

\section{Background}

Psoriatic arthritis (PsA) is an inflammatory arthropathy classified as a seronegative spondyloarthritis characterized by axial and/or peripheral joints damage, it can present recurrent episodes of enthesitis/ dactylitis, skin lesions of psoriasis and other manifestations, such as cardiovascular disease, metabolic syndrome, conjunctivitis, iritis and uveitis [1]. According to various reports, PsA occurs in $6-42 \%$ of patients diagnosed with psoriasis and, in most of the cases, the onset of PsA is concomitant or subsequent to the occurence of psoriatic skin lessions. Thus, psoriasis is considered by some researchers to be a 'prearthritic condition' $[2,3]$.

Important data is gathering for the central role of immune response in the development of psoriasis. Early studies have shown that it is likely that PsA to be mediated by activated $\mathrm{T}$ cells present within early lesional skin [4]; increased cytokine levels of pro-inflammatory IL-15, IFN- $\gamma$, TNF- $\alpha$, and IL-17, are frequently found in psoriatic lesions [5]. Later on, deregulation of innate immunity was reported as involved in this disease and some years ago, the clinical proof was obtained when antiTNF therapeutical approaches (monoclonal antibodies, soluble TNF receptor-Fc fusion proteins) were proven as effective both in psoriasis and PsA [6].

Without early treatment, persistent inflammation in psoriatic arthritis leads to irreversible joint destruction and significant disability with consequent quality of life loss. However, the economical and the psychological aspects of the disease should not be neglected. Thus, a good cooperation between dermatologists and rheumatologists, in order to recognize the early signs of inflammatory arthritis in patients with psoriasis, might be a good strategy for preventing the bad outcome for PsA [7].

In the 'Immunological Disease Continuum' proposed several years ago, PsA stands more on the auto-inflammatory diseases side (Figure 1), PsA comprising forms with and without MHC association. Due to its com-
Constantin Caruntu', ${ }^{1,2}$ Daniel Boda', Georgiana Dumitrascu², Carolina Constantin² \& Monica Neagu*,2

'Dermatology Research Laboratory, "Carol Davila" University of Medicine \& Pharmacy, 22-24 Gr. Manolescu, 0111234, Sector 1, Bucharest, Romania 2"Victor Babes" National Institute of Pathology, 99-101 Splaiul Independentei, 050096, Bucharest, Romania

*Author for correspondence: Immunobiology Laboratory, "Victor Babes" National Institute of Pathology, 99-101 Splaiul Independentei, 050096, Bucharest, Romania Tel./Fax.: +40213194528 neagu.monica@gmail.com 
plex ethyopathology, PsA needs various biomarkers to be validated aiding diagnostic, stratifying various sub-groups and monitoring (immune) therapy efficacy (Figure 1). Spectrum of auto-immune and autoinflammatory diseases portrayed in the 'Immunological Disease Continuum'. Auto-inflammatory diseases can be monogenic inclining toward organ specific, such asPAPA, or being systemic, such asTRAPS, FMF and HIDS. Polygenic autoinflammatory diseases with no MHC association can comprise PsA, while in the mixed pattern diseases Ps and PsA with MHC class 1 association are integrated. In the auto-immunity diseases, the continuum graphic depicts rare monogenic diseases such as ALPS, IPEX and APCED. Polygenic auto-immune diseases with RA in the center, gather a considerable amount of organ-specific or systemic diseases. The graph is not comprehensive by itself, but depicts the complexity of the auto-immune and autoinflammatory diseases (additional information can be found in $[8]$ ).

\section{Diagnosing PsA}

Recent evidence shows that PsA is distinctive from other rheumatic diseases in terms of its clinical, genetic, immunohistochemical and imaging characteristics. Musculoskeletal lesions in PsA comprise peripheral joint involvement, but also include enthesitis and connection of the distal interphalangeal joint. A conventional model for PsA pathogenesis suggests that this is an auto-immune disease, while an alternative model suggests an autoinflammatory pattern for PsA [9]. The histology of psoriasis is characterized by epidermal hyperplasia, dermal angiogenesis and infiltration with mononuclear cells, including activated $\mathrm{T}$ cells and monocytes. Psoriatic arthropathy is associated with skin lesions with the typical appearance of sharply emarginated erythemato-squamous papules or plaques, mainly located on arms, elbows, knees, scalp and trunk. However, skin psoriatic lesions are frequently polymorphic, varying in aspect and size and require histological evaluation to confirm the clinical diagnosis $[13,14]$.

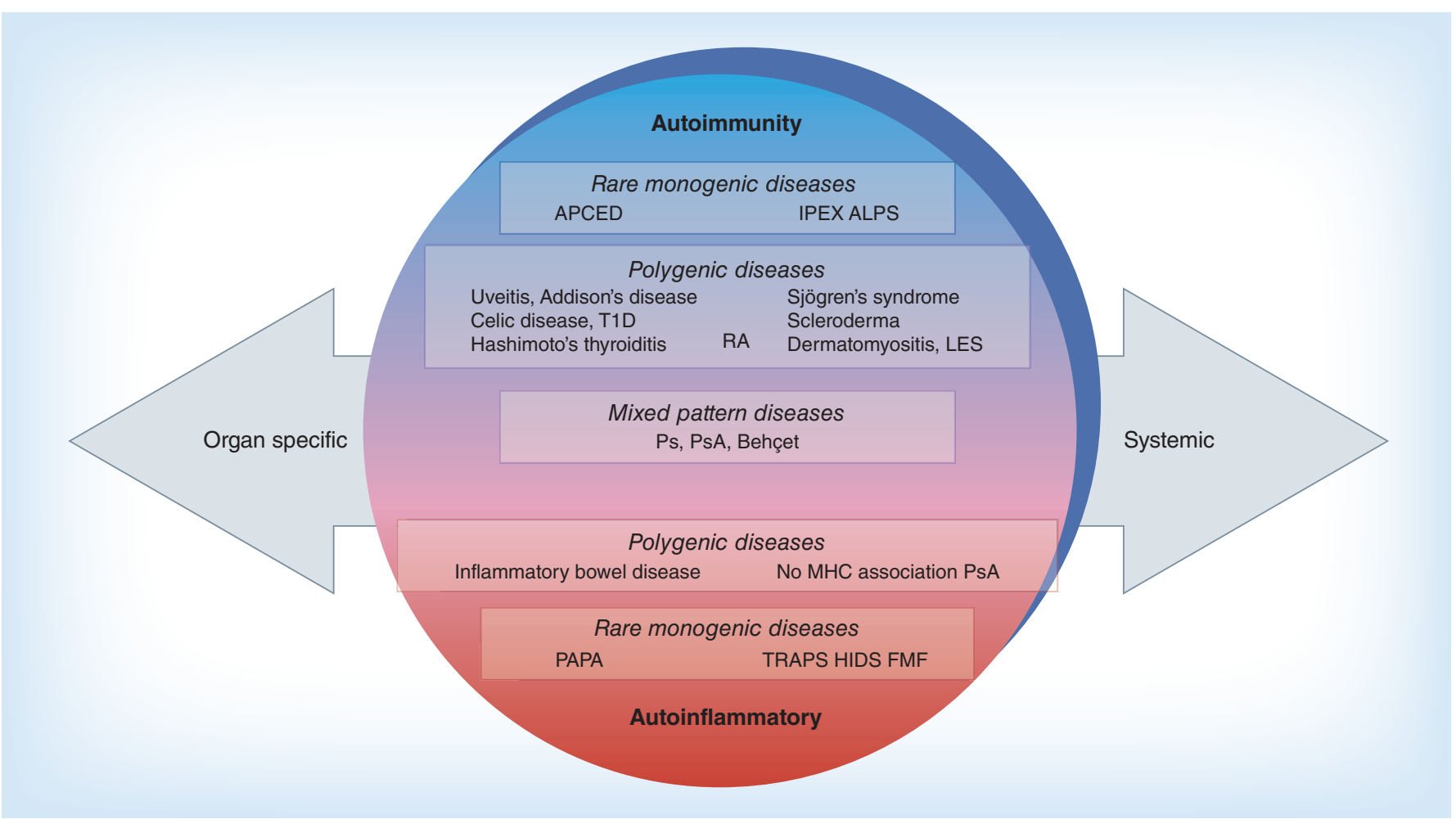

Figure 1. Spectrum of auto-immune and autoinflammatory diseases portrayed in the 'Immunological Disease Continuum'. Autoinflammatory diseases can be monogenic inclining toward organ specific, such as PAPA, or being systemic, such as TRAPS, FMF and HIDS. Polygenic autoinflammatory diseases with no MHC association can comprise PsA, while in the mixed pattern diseases Ps and PsA with MHC class 1 association are integrated. In the auto-immunity diseases, the continuum graphic depicts rare monogenic diseases such as ALPS, IPEX and APCED. Polygenic auto-immune diseases with RA in the center, gather a considerable amount of organ-specific or systemic diseases. The graph is not comprehensive by itself, but depicts the complexity of the auto-immune and auto-inflammatory diseases (additional information can be found in [8]).

ALPS: Auto-immune lymphoproliferative syndrome; APCED: Auto-immune polyendocrinopathy-candidiasis-ectodermal dystrophy; FMF: Familial Mediterranean fever; HIDS: Hyperimmunoglobulinemia D Syndrome; IPEX: Immunodysregulation polyendocrinopathy enteropathy X-linked syndrome; LES: Systemic lupus erythematous; PAPA: Pyoderma gangrenosum and acne; Ps: Psoriasis; PsA: Psoriatic arthritis; RA: Rheumatoid arthritis; T1D: Type 1 diabetes; TRAPS: TNF receptor-associated periodic syndrome. 
The histological representation of cutaneous psoriasis lesions include increased thickness of the stratum corneum, incomplete keratinocyte maturation with parakeratosis, acanthosis with elongation and thickening of the rete ridges associated with suprapapillary epidermal thinning, diminishing or absence of granular layer, presence of characteristic spongiform pustules with aggregates of neutrophils in the spinous layer or collections of neutrophils within parakeratotic areas of the cornified layer resulting Munro micro abscesses, elongated dermal papillae with tortuous, dilated capillaries and an inflammatory cell infiltrate in the upper dermis. Modern imagistic techniques can provide in depth characterization of tissue abnormalities. Reflectance confocal microscopy (RCM) is a high-resolution imaging technique that facilitates a real time assessment of cell morphology in epidermis and superficial dermis. It is an easy and fast procedure that allows a non-invasive morphological and functional evaluation of multiple skin lesions unlimited in time [11]. According to recent studies, by using RCM it is possible to identify in vivo most of the typical histological features of psoriasis (see Figure 2), thus demonstrating a promising link between the RCM and routine histology [12].

The epidermal hyperplasia that is a major characteristic of the disease may be due to an increased number of cycling cells in the basal layer of the epidermis caused by a decrease in apoptosis or an increase in the rate of cell division [5]. Studies testing this proliferative capacity have shown that in vitro performed cell proliferation induced by cytokines and growth factors are not different between keratinocytes isolated from normal versus psoriatic skin [13]. Our studies, though, have shown that there is an increased proliferation rate of keratinocytes isolated from psoriatic lesions. We have shown that isolating keratinocytes after prior standardized protocols from $3 \mathrm{~mm}$ diameters skin punch biopsies, their proliferation capacity is at least double in comparison with keratinocytes isolated from normal skin (Figure 3) [14].

In the quest of identifying imagistic features of psoriasis and PsA, a recent study has been developed using ultrasonography with power Doppler (PD). The aim of the study was to depict the 'inflammatory' or vascular phenotype in psoriasis and PsA. Using PD, a more inflammatory or vascular process was identified in PsA patients, that can further be linked when the disease progresses from skin to joint [15]. Multi-level approaches, from immunological and molecular domains to imagistic and magnetic resonance field, thoroughly studied by the 'Group for Research and Assessment of Psoriasis and Psoriatic Arthritis' (GRAPPA), have drawn some seminal conclusion. Thus, PsA has a central backbone comprising inflammatory cascades and cytokine net- works that are converging to a sustained inflammation leading to altered tissues; moreover, this important extended group ralies important research focus group in validating new biomarkers [16].

\section{Future diagnostic biomarkers}

Although intensively studied, there is still an unmet need of detecting screening and early biomarkers for PsA. It has recently been shown that out of over 300 plasma proteins [17] in PsA and RA patients, several proteins have been found significantly modified compared with controls; while some were up-regulated (e.g., complement factor I, complement component C8- $\beta$, glyceraldehyde-3-phosphate dehydrogenase and inter$\alpha$-trypsin inhibitor heavy chain H1), other were downregulated (immunoglobulin heavy chain V-III region $\mathrm{BRO}$, and immunoglobulin J chain). The only proteins that are proposed by the authors as future diagnostic biomarkers, depicting PsA versus RA are Serpin A11, complement factor $\mathrm{H}$-related protein 5, cartilage acidic protein 1 and coagulation factor IX). These four proteins were found down-regulated in PsA, while found up-regulated in RA patients. Another recent study showed auto-antibodies as possible plasma biomarkers in PsA. As autoantibody's presence is not a characteristic of PsA, finding this type of immune molecule can stratify PsA patients in various risk sub-groups [18]. Antibodies that are generated against citrullinated vimentin (anti-MCVs) were one of the auto-antibodies reported as associated with PsA [19]. In PsA patients that are displaying clear symptomatology, the anti-MCV serum concentration was higher than in the group without any joint symptoms. When finding higher levels of anti-MCV in the patients without join involvement, the clinical course of the disease was more severe. Therefore, these antibodies can present future biomarker predictive power in stratifying patients with good and bad clinical outcome. Incorporating 'classical' biomarkers, such as the CRP protein, in a panel comprising several other proteins can enhance the biomarkers armentarium in PsA. The group of Chandran has shown that the combination of CRP with osteoprotegerin (OPG), matrix metalloproteinase 3 (MMP-3) and the ratio of C-propeptide of Type II collagen (CPII) to collagen fragment neoepitopes Col2-3/4(long mono) (C2C) can be considered a screening biomarkers' panel for PsA [3]. Another panel of soluble biomarkers, comprising MMP3, hs-CRP and VEGF could constitute a future early detection biomarkers panel for PsA, to be further used in order to monitor disease progression [20].

Diagnostic biomarkers in PsA are still in their early stages, needing validation on large cohorts of subjects. Immune markers, key biomarkers in PsA, can be tissue and/or soluble types, wether per se immune mark- 

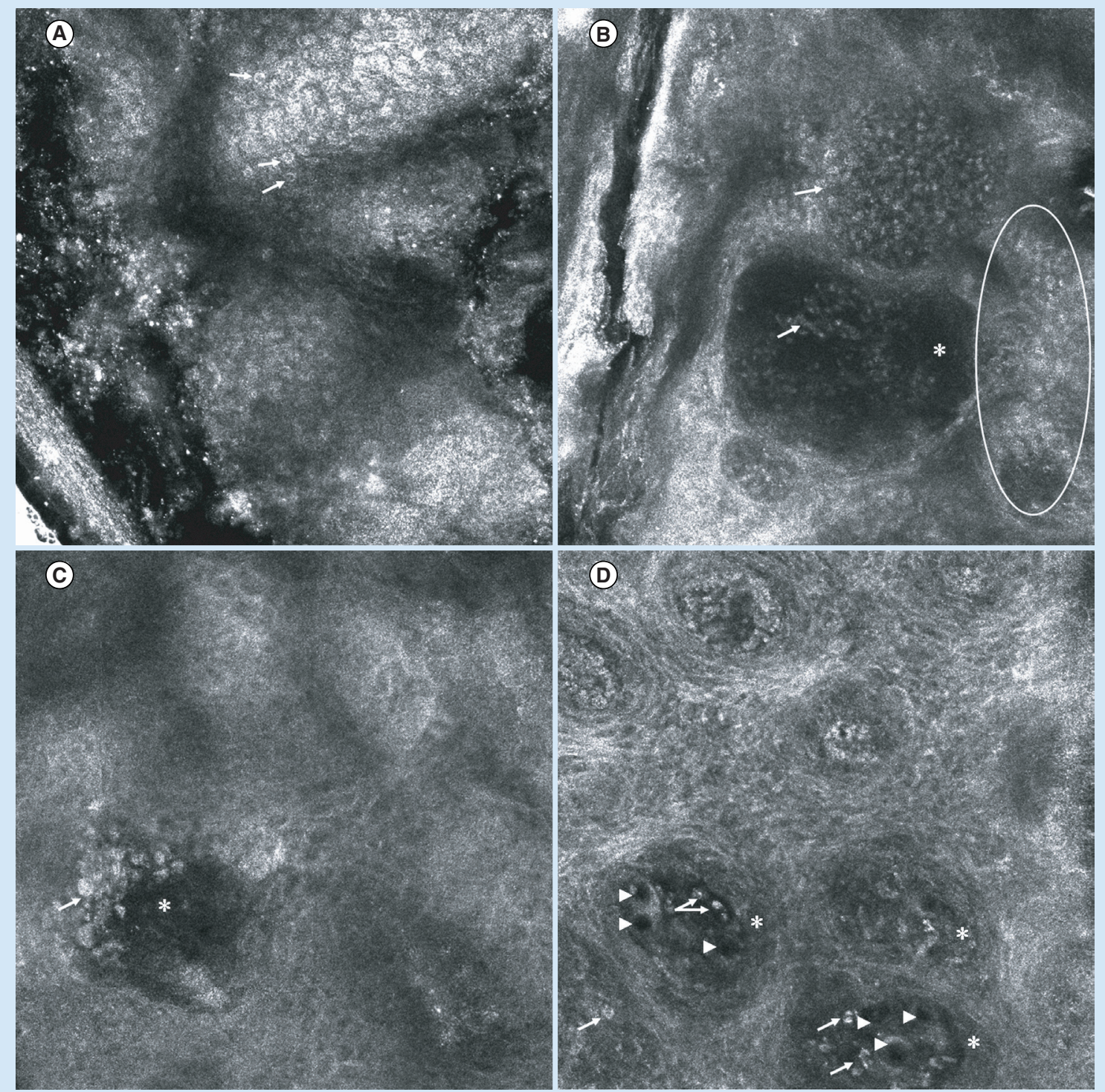

Figure 2. Reflectance confocal microscopy $(\mathrm{RCM})$ images $(500 \times 500 \mu \mathrm{m})$ of psoriasis skin lesions. (A) Numerous, round to polygonal, nucleated bright structures $(\rightarrow)$ are detected in the cornified layer corresponding to parakeratotic keratinocytes and/or inflammatory cells. (B \& C) Refractile round to polygonal structures clustered together $(\rightarrow)$ corresponding to accumulation of infiltrating leukocytes may be visualized in the parakeratotic areas (white circle) of the cornified layer (Munro micro abscesses) or in the upper portion of the spinous layer, where they are associated with dark areas $\left(^{*}\right)$ in the honeycombed structure of epidermis (spongiform pustules of Kogoj). (D) The dermal papillae (*) are enlarged and are visible above the dermo-epidermal junction. Their density is increased, they lack the bright ring of basal cells while the thin surrounding epithelial tissue has an irregular honeycomb pattern. Dilated and tortuous capillaries are visible as dark round structures $(\boldsymbol{\Delta})$ inside the papillae; bright round structures $(\rightarrow$ ) corresponding to inflammatory cells may be detected in the papillary dermis, around the capillary vessels and in the epidermis.

ers (e.g., auto-antibodies or complement molecules) or immune-related ones (e.g., MMPs or VEGF).

\section{Immune mechanisms in the psoriatic arthritis pathogenesis}

Immunopathology and immune diagnostics have important words to say in PsA, a disease that can be generated by an external trigger (e.g., mechanical stress, trauma and infection) in genetically susceptible patients. As further depicted in this section, the immune-mechanisms that are hypothesized for PsA generation can furnish future biomarkers. A schematic view regardind PsA immunopathogenesis is presented briefly in Table 1.

In psoriatic lesions there is an increased number of activated phenotype dendritic cells (DCs) [27,31] that 


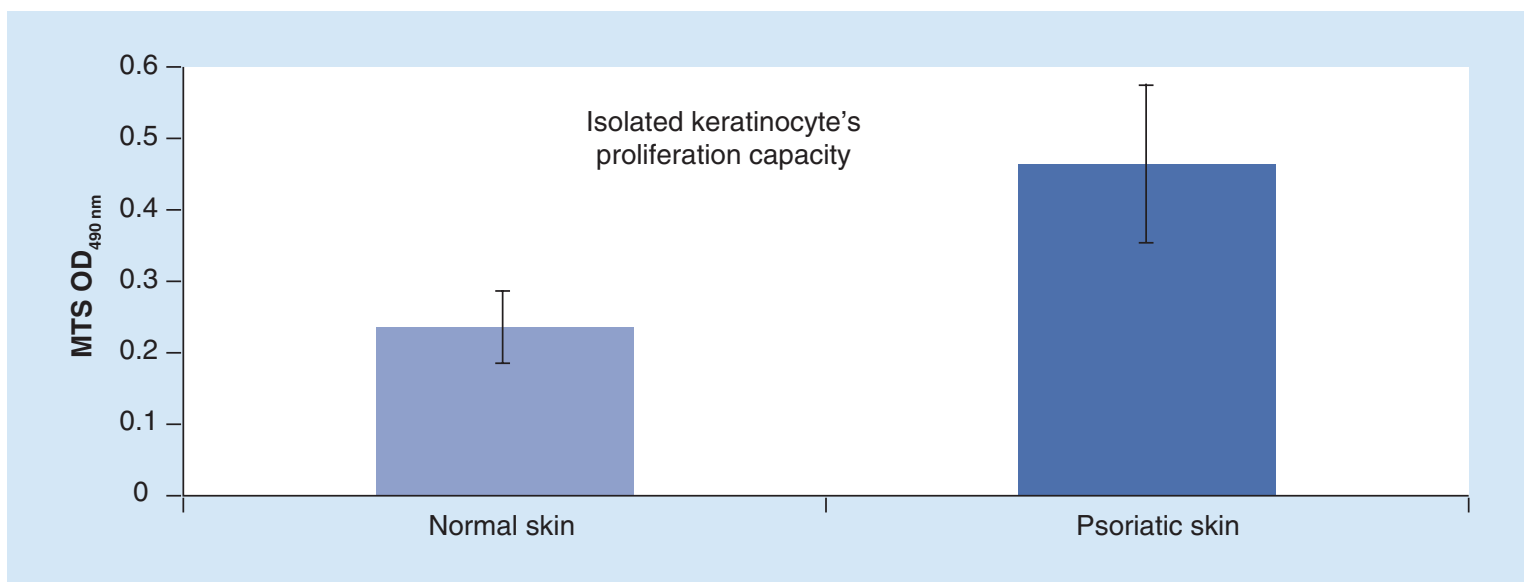

Figure 3. Proliferation capacity of isolated keratinocytes from normal and psoriatic skin after $24 \mathrm{~h}$ culture, evaluation with MTS [14].

MTS: (3-(4,5-dimethylthiazol-2-yl)-5-(3-carboxymethoxyphenyl)-2-(4-sulfophenyl)-2H-tetrazolium).

activate resting cells Th-1 subtype [21]. More than 10 years ago, in psoriatic lesions, several proinflammatory cytokines were found up-regulated, including IFN- $\gamma$ and TNF- $\alpha$, along with up-regulation of IL-15 and its receptor. All these cells contribute to the released cytokines that are highly involved in the pathologic changes induced in lesional keratinocytes and in vascular endothelium [22].

Recognizing that PsA has two target organs (skin and joints) opened a new window in deciphering the immunopathophysiology of PsA. The recently reported Th-17/IL-23 immune axis has been shown to be involved in both psoriasis and PsA. Moreover, it was highlighted that a trigger for psoriasis and PsA can be an environmental factor that raises the natural immunity response [23]. Th-17 cells are a relatively new described type of Th cells producing IL-17A, IL-17F, IL-21 and IL-22, being functionally different from the 'classical' Th1 and Th 2 cells. This new sub-population of Th has been reported to be involved in acquired immune responses against bacterial, mycobacterial and fungal pathogens, being further at the initiation of several auto-immune diseases [24].

Abnormal innate and adaptive immune activation, as an initial response to an external trigger, may lead to the chronic disease stage based on genetic predisposition [25]. The diagram presented in Figure 4 shows a possible mechanism that initiates psoriasis and PsA.

The complexity of PsA's pathophysiology is due to the fact that immune cells and molecules are residing in different tissues like the synovium, skin and axial joints [26]. Pro-inflammatory cytokines (IL-12/IL-23) drive the development of autoreactive $\mathrm{T}$ lymphocytes actively producing IL-17. A chronic inflammation milieu is generated by innate immunity cells, neutrophils and monocytes, surrounded by high concentrations of IL-17, IL-6, IL-8 and TNF. Therefore, in the recent years, in the context of auto-immune inflammation perpetuation, the generated immune response based on IL-23-IL-17 pathway was highlighted [26].

Dendritic cells (DCs) from PsA patients have an abnormal functionality that can impose chronic inflammation upon an initial trigger encounter and recent findings have shown that upon in vitro stimulation with mycobacterium and TLR-2 ligands, high levels of C-reactive protein emerge [27]. Suppressor of Cytokine Signaling 3 (SOCS-3) is part of a larger family that sustains a negative feedback system for cytokine signal transduction. When analyzing DCs from PsA patients, SOCS-3 and A20 (a tumor necrosis factor TNF- $\alpha$-inducible zinc finger protein involved in the NF- $\kappa \mathrm{B}-$ mediated immune responses) were highly expressed. Meanwhile these DCs had an up-regulated level of ATG16L1, NADPH oxidase 2 and LL37 (the active peptide LL-37 of cathelicidin cationic antimicrobial protein-18), molecules with recognized immune effect against intracellular bacteria. This potential impairment of immune response sustained by one of the main cells that are clearing microbes can contribute to the setting-up of the chronic inflammation that affects the joints, entheses, skin and gut [25,27].

As reviewed by Lloyd et al. initially the increased number of IFN- $\gamma$ producing cells found in cutaneous eruptions from psoriasis and PsA conducted to the hypothesis of Th1-cell mediated disorders [1]. More recent studies highlighted the pivotal role of Th-17 in the pathology of both psoriasis and PsA [28]. Th-1 and Th-17 cells are differentiated after stimulation of myeloid dendritic cells by TNF- $\alpha$, IFN- $\gamma$, IFN- $\alpha$, IL- 6 and IL-1 $\beta$, DC stimulation induces IL-12 and IL-23 secretion [1]. IL-12 stimulates Th-1 differentiation with consequent increased production of IFN- $\gamma$, whereas IL-23 stimulates Th-17 cells, which induces the synthesis of the pro-inflammatory mediators such 


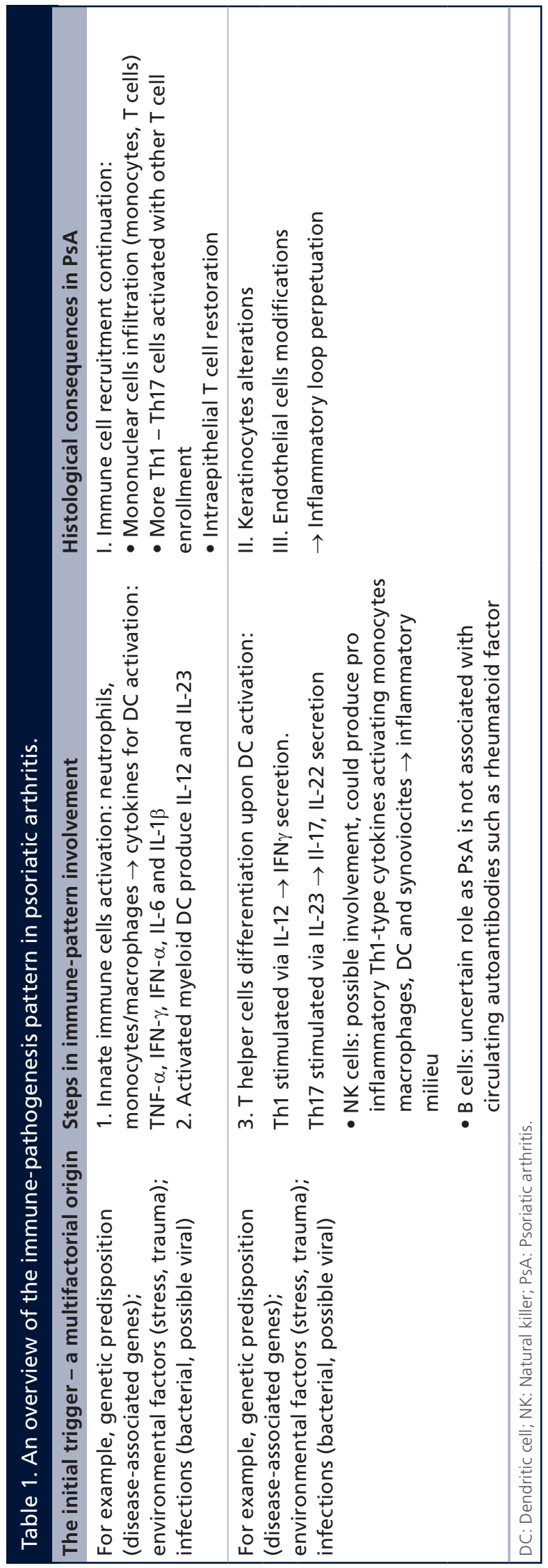

as IL-17 and IL-22 [29]. Histologically, PsA has diffuse infiltrate of $\mathrm{B}$ and $\mathrm{T}$ lymphocytes, macrophages, neutrophils and DCs. It seems that $\mathrm{T}$ lymphocytes have specific clones for both skin and synovium. The skin lesions abound in monocyte-derived cytokines expression, namely S100A8/A9 [30]. In relation to the increased expression of tissue S100A8/9, another group found that these molecules are increased in the PsA patients' serum. Comparing several diseases, such as psoriasis vulgaris, PsA, pustular psoriasis and atopic dermatitis, it was reported that patients have increased serum values compared with controls. The authors report that the highest serum level was obtained in patients developing articular involvement without correlation with the affected skin surface. These two molecules have different origin; while S100A8 is expressed by keratinocytes and infiltrating mononuclear cells, S100A9 is secreted by neutrophils. The high level of circulating S100A8/9, reside in the overall activated monocyte/macrophage lineage [30].

There are very few data regarding complement components in PsA patients, but lately increased plasma levels of C3 and C4 complement components in PsA patients were reported. This is a good argument for the possibility hinted a few years ago, that the interaction between TNF $\alpha$ and complement system could trigger this pathology. Moreover, TNF $\alpha$-inhibitors targeting complement components can be future therapy targets in PsA [31].

For the vast majority of inflammatory CC chemokines (CC chemokine ligand 2 [CCL2], CCL3L1, CCL4, CCL5, CCL7, CCL8, CCL11, CCL13, CCL14, CCL17 and CCL22), D6 is known as their high-affinity receptor [32]. It has been recently shown that D6 is involved in controlling skin inflammation; moreover, D6 deficiency can participate to the human psoriatic skin pathology. The reported data show that elevated D6 levels suppress inflammatory chemokines and hence skin lesion development. Interestingly, D6 was found elevated in peripheral blood leukocytes in PsA patients, as a systemic response to inflammation [33]. In the synovial fluid collected from PsA patients, a significantly higher concentration of macrophage-derived chemokine (MDC/CCL22) was found, level that was in correlation with the increased plasmatic level found in the same patients. The authors show that this sustains a migration mechanism of CCR4 expressing memory cells, probably attracting $\mathrm{T}$ cells from the skin to the joints and a possible biomarker for an evolving pathologic condition [34].

As the initial trigger can activate NK cells, these cells were also studied as being involved in the immunepathogenesis of PsA. Particular allotypes of the killer 
cell immunoglobulin-like receptors (KIR) superfamily were seen in NK cells isolated from PsA. NK can produce pro-inflammatory Th1-type cytokines, activating monocytes, DCs and synoviocites, thus maintaining an inflammatory milieu [35]. An interesting involvement of NK in PsA's pathogenesis was recently flagged. It is known that CXCL10 can be secreted by $\mathrm{CD}^{+} / \mathrm{CD}^{+}$ $\mathrm{T}$ lymphocytes, but also by NK and NK-T cells, this secretion being induced via IL-12. The possibility to quantify CXCL10 in peripheral blood and in the synovial fluid can indicate a new immune marker in PsA [36]. In the inflamed skin, recruited Th-1 cells secrete IFN- $\gamma$ and TNF- $\alpha$, stimulating further more CXCL10 secretion and inducing an auto-immune loop perpetuation. Thus, a recent study suggested CXCL10 as a possible biomarker candidate and a new therapy target [37]. A few years ago it was reported that in chronically inflamed tissues a distinctive NK subset with the phenotype $\mathrm{CD} 3^{-} \mathrm{CD} 56^{\text {bright }}$ can accumulate. This subset induces $\mathrm{CD} 14^{+}$monocytes to differentiate into DCs that vigorously activate Th1 lymphocytes. Synovial fluid from PsA patients triggers monocytes differentiation in DCs, only in the physical presence of NK. It is one of the first reports highlighting the important role of NK in maintaining a local inflammation in PsA through DCs differentiation pathway and the fact that the presence of this distinctive NK subset can mark the appearance of an auto-inflammation pathological process [38].

DCs involvement in PsA have their 'fair share' in the immune pathogenesis of this disease. Peripheral blood plasmacytoid DCs (pDCs) are found significantly reduced in PsA patients, having a somewhat immature phenotype with reduced L-selectin (CD62L) expression. In PsA's patients synovial fluid, both myeloid DCs (mDCs) and $\mathrm{pDCs}$ were found. mDCs from the synovial fluid displayed a more mature phenotype with an increased expression of CD80, CD83 and CD86, maturation that seems to be mediated through TLR stimulation [39].

Studying TLR-2 in APCs from PsA, it has been shown that TLR-2 expression is increased in immature DCs, while monocytes and mature DCs are not different from the control group, with no difference for TLR-4 expression. In in vitro cell studies, cell's supernatants present high concentrations of Th1 type cytokines, TNF- $\alpha$, IFN- $\gamma$ and IL-2. The authors state that TLR-2 expression reported in this study can account for the immune-pathogenesis of PsA [40].

CD40L binds to the CD40 that is expressed on APC, being a costimulatory molecule. On T cells, CD 40L is an early activation marker and its expression was found significantly increased on $T$ lymphocytes from PsA patients [41]. In search of immune biomark-

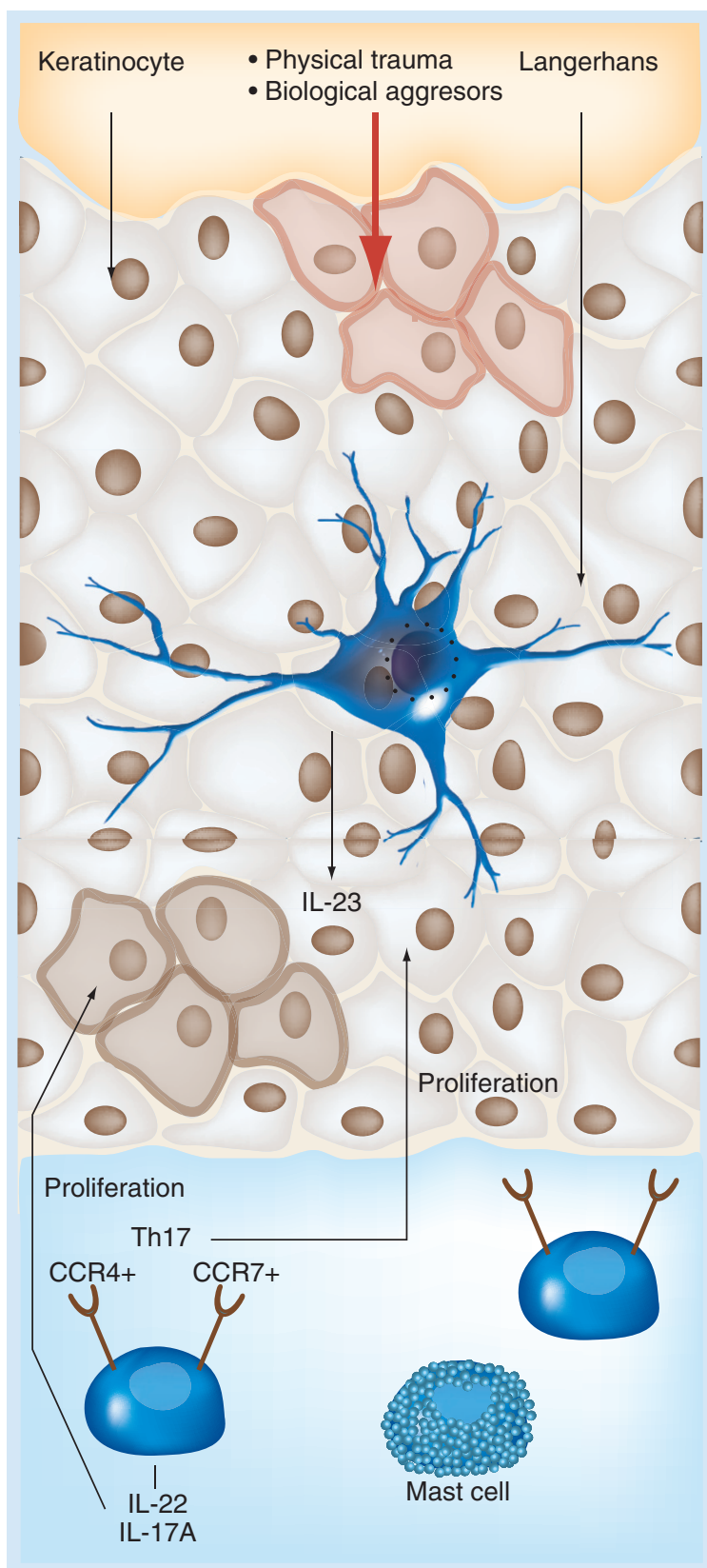

Figure 4. Initial generation of pro-inflammatory cytokines in skin subjected to physical and/or biological trauma. When keratinocytes are injured they generate along with dendritic cells (Langerhans cell), IL-23. This cytokine induces proliferation of Th17 CCR4+ and CCR7+, cells that secrete among others, IL-22 and IL-17A; these cytokines act as growth factors upon keratinocytes that are induced to proliferate.

ers, it has recently been reported that in the serum of PsA patients, soluble CD40 and CD40L were found increased in comparison to controls. No significance was reported between these circulating molecules and the duration and presence of PsA symptoms [42]. In Table 2 is depicted an outline of cells and corresponding cytokines involved in PsA. 
Table 2. Cytokines and chemokines in psoriatic arthritis.

\begin{tabular}{|c|c|c|}
\hline Cytokine & Cellular type source & Function in PsA \\
\hline IL-12/IL-23 & Monocytes, macrophages, DC & $\begin{array}{l}\text { Driven the development of autoreactive Th1 } \\
\text { and Th17 cells } \rightarrow \text { auto-immune inflammation }\end{array}$ \\
\hline II-6, IL-8, TNF $\alpha$, IFNs & $\begin{array}{l}\text { Monocytes, macrophages, T cells, } \\
\text { endothelial cells }\end{array}$ & Chronic inflammatory milieu maintaining \\
\hline IL-17 & $\begin{array}{l}\text { Neutrophils, mast cells, } \\
\text { autoreactive T cells }\end{array}$ & $\begin{array}{l}\text { Driven the development of autoreactive Th17 } \\
\text { cells } \rightarrow \text { auto-immune inflammation }\end{array}$ \\
\hline SOCS-3 & DC & Setting-up the chronic inflammation \\
\hline TNF- $\alpha$, IFN- $\gamma$, IL-2 & DC & Account for immune-pathogenesis \\
\hline Soluble CD40/CD40L & DC & Account for immune-pathogenesis \\
\hline S100A8 & $\begin{array}{l}\text { Infiltrating monocytes } \\
\text { Keratinocytes }\end{array}$ & Activation of Mo/Mf in skin lesions of PsA \\
\hline S100A9 & Neutrophils & Activation of Mo/Mf in skin lesions of PsA \\
\hline D6 chemokines & Blood leukocytes & Controlling skin inflammation \\
\hline CXCL10 & NK cells & $\begin{array}{l}\text { Contribute to auto-immune loop, candidate } \\
\text { for new therapy target in PsA }\end{array}$ \\
\hline
\end{tabular}

Immune-pathogenetic mechanisms in PsA still bear a reservoir of future biomarkers candidates important for monitoring the evolution of the disease and unveiling new therapy targets.

\section{Biomarkers associated with psoriatic arthritis}

\section{Genomic biomarkers}

In the last years, a common agreement has been reached upon the fact that PsA has a genetic background. Focusing on single nucleotide polymorphisms (SNPs), the most important association with PsA was found to be $M H C I$ gene. Another association was found between $I L 23 R$ region and a SNP 4 $\mathrm{kb}$ upstream from IL12RB2. The region harboring ubiquitin-specific protease- 8 lies upstream from $H L A-C$, accommodates as well the gene for SPPL $2 A$ (signal peptide peptidase such as $2 \mathrm{a}$ ). SPPL2A activates TNF- $\alpha$ inducing in DCs IL12 expression. All these genetic markers are associated with auto-immune diseases [43]. A very recent study shows that, in Romanian population, exonic SNP rs11171806 of the IL23A gene was found under-expressed in PsA, while the carriers of $r s 11171806 / r s 2066808 A C$ haplotype had a low risk of developing PsA. The $I L 12 B$ gene and its variants were found associated in patients diagnosed with polyarticular PsA [44].

Psoriasis that has a $H L A-C w 6$-association is linked to auto-immunity, in contrast to the joint disease form that can be triggered more by the tissue microtrauma, leading to auto-inflammatory immune responses. The assertion that immune-pathology of psoriasis and of
PsA can have different immune arms involvement, adaptive and innate immunity, strengthens once more the position of this disease along the 'immunological disease continuum' [45] (see also Figure 1).

GRAPPA has stated that $H L A-C$ is a PsA gene, having $H L A-C w^{*} 0602$ as risk allele, thus recently other genes entered the genomic biomarkers candidates [46]. $H L A-B^{*} 27, H L A-B^{*} 38, H L A-B^{*} 39$ and $H L A-C^{*} 12$ alleles are potential PsA-specific genetic markers among patients with psoriasis [47]. A study performed on over 600 patients, published this year, has shown that KIR2DL2 and KIR2DS2 polymorphisms are significantly associated with the disease [48]. In other extended study, REL gene, (a gene involved in NFKB associated mechanisms), is associated with PsA and that there are different alleles in RA and PsA depicting basic genetic differences in these somewhat 'overlapping' diseases [49].

Moreover, genes from IL-23/IL-17-mediated lymphocyte signaling pathways were found linked to PsA [46]. Lately, it has been reported that the profile of genetic susceptibility differs in European and Chinese populations. Even in this recent paper, the reported loci do not account for the PsA susceptibility, leaving room to study the genetic - environmental factors interaction that leads to the development of PsA's full blown pathology [50].

All these recently reported genes can be part of cutaneous signaling networks and interferon signaling pathways and can stand for genetic biomarkers, depicting the susceptibility of an individual to develop this form of auto-inflammatory disease (Table 3). 


\section{Proteomic technologies \& proteomic biomarkers}

Similar to other pathologies, personalized medicine could be an important approach in inflammatory arthritis through the potential of the '-omics' technologies to deliver new biomarkers for early diagnosis, for identification of patients predisposed to a bad prognosis and for selection of the therapy-responders. The increasing interest of proteomics in the field of rheumatology highlighted a few years ago that biomarkers need to differentiate "between different rheumatic disease subtypes like rheumatoid arthritis and spondyloarthropathy" [51]. To our knowledge, there are only a few studies focusing on proteomics in PsA, in contrast to the surge of biomarkers discovery in RA. Recently, a novel multiplex technology IMPACT (Immunological Multi-Parameter Chip Technology) was put in use for differential diagnosis between RA and other inflammatory arthritis and for defining the clinical stages of RA at molecular level. This recent study aimed to measure auto-antibody reactivity's and bone-turnover markers in serum samples from patients with RA, with ankylosing spondylitis and with PsA. Results have shown that a panel of six autoantibodies (histone $2 \mathrm{~B} / \mathrm{e}$, vimentin, fibrinogen A, COMP, profilaggrin, RF-IgA) was significantly higher in RA patients than in ankylosing spondylitis patients, in PsA patients or in healthy controls. Thus, multiplex-biomarker detection could be an important tool for rheumatologists since it provides high sensitivity ( 84.2 or $59.2 \%$ ) and specificity ( 93.8 or 96.3\%) in RA diagnosis when three, respectively four of these biomarkers are positive. A larger panel of autoantibodies is recommended in order to correct any misdiagnosis due to the unusual detection of autoantibodies in several patients diagnosed with PsA [18].

Another proteomic approach for identifying serum biomarkers of arthritis is represented by SELDI-TOF MS technology. Thus, in 2008, using this technology, several proteins were investigated, S100A8 (calgranulin A), S100A9 (calgranulin B), S100A12 (calgranulin C), SAA (serum amyloid A), SAA-R (SAA des-Arg), SAARS (SAA des-Arg/des-Ser) in RA, PsA, ankylosing spondylitis patients and patients with inflammatory bowel diseases. The study found out that SAA, SAA-R and SAA-RS could distinguish all inflammations (except spondylitis) from non-inflammatory control group and that $S 100$ proteins could differentiate the inflammatory arthritis (RA, PsA and spondylitis) from non-inflammatory control group and from inflammatory bowel diseases. A slight difference regarding S100A proteins in PsA and spondylitis patients was noticed, whereas S100A12 significantly distinguished RA patients from PsA patients [52].

Also, there are a few studies in literature that use proteomic technologies in psoriasis, investigating subgroups with and without arthritic component. Recently, some soluble serum potential biomarkers could differentiate patients with PsA from those with cutaneous psoriasis alone: highly sensitive CRP (hsCRP), osteoprotegerin (OPG), MMP-3 and the C-propeptide of Type II collagen (CPII): collagen fragment neoepitopes Col2-3/4long mono (C2C) ratio. It has been suggested that a profile of soluble biomarkers in psoriasis compared with PsA can furbish an important tool for early stratification of patients on the bridge of developing the arthritic form and thus identifying a worse clinical outcome [53].

Proteomic analysis using 2D gel electrophoresis and LC-MS/MS analysis revealed an up-regulated PRDX2, GSTP1 and SFN in psoriatic skin tissue. It seems that massive infiltration of leukocytes in psoriasis can lead to a local release of pro-oxidative species involved in the proinflammatory activation of keratinocytes and fibroblasts. Up-regulated GSTP1 and PRDX2 in psoriasis may be explained by their key roles in preventing cells from ROS-induced DNA

Table 3. Genes linked to psoriatic arthritis susceptibility.

\begin{tabular}{lll}
\hline Genes & Involved in & $\begin{array}{c}\text { Ethnicity } \\
\text { predisposition }\end{array}$ \\
\hline$H L A-C W^{*} 0602$ risk allele & HLA-C & No \\
\hline$L C E 3, D E F B 4, G J B 2$ & Cutaneous signaling networks & - \\
\hline TNFAIP3, TNIP1, NFKBIA, REL, FBXL19, TYK2, NOS2 & Interferon signaling pathways & - \\
\hline IL12B, IL23R, IL23A, TRAF3IP2, ERAP1 & IL-23/IL-17-mediated lymphocyte & - \\
& signaling & European \\
IL13, IL4, TNFAIP3, IL23A, IL23R, IL28RA, REL, IFIH1, & Inflammatory processes & \\
ERAP, TRAF3IP2, NFKBIA, TYK2, ZNF313, NOS2, & & Chinese \\
FBXL19, NFKBIA & & \\
\hline IL12B, LCE3D, ERAP1, TNIP1, PTTG1, CSMD1, GJB2, & Inflammatory processes & \\
SERPINB8, ZNF816A & &
\end{tabular}


damage or cell death [54]. Our experience has shown that phagocytes release in the synovial fluid of RA patients various ROS and maintaining thus an oxidative stress both in periphery and in the inflamed joint [55]. We do not rule out that as in RA, in PsA's affected joints there is an oxidative environment that sustain as well inflammation.

Analyzing skin's proteomics, it has been reported that the up-regulated SFN (14-13-13 protein $\sigma$ ) found in the skin of PsA patients, a protein known to be involved in the cytoplasmic sequestration of the proapoptotic protein (Bax), may be involved in preventing apoptosis and promoting epidermal hyperplasia in psoriatic cells [54].

Combining MALDI-TOF mass-spectrometry and 2D electrophoresis for lesional skin biopsies in PsA, 10 proteins with at least 2 -fold increased expression in lesional skin compared with nonlesional skin were found. These proteins (Keratin 14, Keratin 16, Keratin 17, Squamous cell carcinoma antigen, Squamous cell carcinoma antigen-2, Enolase 1, Superoxide dismutase, Galectin-7, S100A9 and S100A7) were associated with overexpressed encoding genes (seven out of ten protein markers). These findings prove a substantial overlap between transcriptomics and proteomics data in PsA [56].

Recently, Dalmady's group brought evidence for significantly higher anti-MCVs (antibodies targeting mutated citrullinated vimentin) in serum of PsA patients compared with psoriasis patients with/without joint symptoms. The authors could not demonstrate a direct relation between the anti-MCV titers and enthesopathy scores, but they could establish a relation between nail psoriasis and the presence of anti-MCV. Thus, it was suggested that high anti-MCV level may be a marker of systemic enthesitis in PsA, since in previous studies, enthesopathy scores were significantly higher in PsA patients with nail psoriasis than in those without it [19].

Other biomarkers reported to distinguish between Psoriatic Arthritis and Psoriasis are: Dickkopf-1 (DKK-1), macrophage-colony stimulating factor (M-CSF), highly sensitive CRP (hsCRP), osteoprotegerin (OPG), MMP-3 and the C-propeptide of Type II collagen (CPII) [3].

Considering the 'massive erosion', aggressive subtypes (with the most severe form - arthritis mutilant) and 'unpredictable responses to therapy', multiplex biomarkers assays should be better put to work in order to provide a panel of multiple protein biomarkers for early diagnosis of arthritis component in psoriasis patients, for differential diagnosis between different PsA subtypes and for identifying therapy responders [57].
Thus, proteomic technologies should be used on larger groups of PsA, not only compared with other rheumatic diseases for differential diagnosis, but also within the group for establishment of the relation between different subtypes of PsA.

Taking into account our experience in proteomic biomarker discovery on other pathologies, we can conclude that this field has a huge potential in PsA. Consequently, we suggest that multi-proteomic approaches like protein microarray technology that can bring important information for parallel evaluation of biomarkers in circulation and at the site of auto-immunity/ auto-inflammation tissues.

\section{Animal models: important tools in psoriatic arthritis preclinical studies}

The need of meaningful animal model in this disease has rose mainly from the preclinical studies that tried to depict intimate pathogenic mechanisms and pinpoint new therapy targets. Animal models in PsA ranged from rodents to minipigs and were used from xenotransplantation approaches to knock-down models, and spanned more than two decades of development.

In the psoriasis xeno-transplantation model in SCID mice, 10 years ago, it was shown that the local tissue immune response is dominated by IFN- $\gamma$ and IL-15 expression. This was one of the first animal models studies proving that Th1 lymphocytes and NK cells can be involved in the full-fledged inducement of psoriatic plaques [58]. In a mouse xenograft model for human psoriasis, the deregulation of IL-15 and its appending signaling pathway has been demonstrated as being involved in pathogenesis. IL-15, having structural similarity to IL-2, is secreted mainly by mononuclear phagocytes upon virus infection. The link with other cells is realized by the fact that this is a cytokine that induces NK activation and proliferation, hence the recently proven NK increase [59]. In IFN- $\gamma$-deficient AGR mice, when xenotransplanting unaffected skin of PsA patients, the disease can be initiated. The initiation of disease is mediated by the alphalbetal integrins that control Th1 memory cells in the epidermis [60].

Wounded skin of transgenic K5.Stat3C mice comprise keratinocytes that express several up regulated molecules linked to the pathogenesis of psoriasis. These lesions are developing toward psoriatic type when Stat3 is activated in keratinocytes that further induce $\mathrm{T}$ lymphocyte activation. This is another model reinforcing $\mathrm{T}$ cell involvement in lesion development [61]. In a further mouse model, Cd18 hypomorphic PL/J (Cd18hypo), a model mimicking human psoriasis has demonstrated that low expression of $\beta 2$ integrin impaired the tandem Tregs DCs cellular interaction. This lack of efficient immune inter-cellular interaction did not suppress pathogenic $\mathrm{T}$ 
cells, in a TGF- $\beta$-dependent way. These data published several years ago have shown once again Treg involvement in psoriasis [62]. Several years were needed for animals models to reach a PsA model; therefore, last year, a mice model of doxycycline-inducible human TNF- $\alpha$-transgenic mice was reported; it can develop inflammatory arthritis- and psoriasis-like phenotype. The existence of this new model can be utilized for early phases of development; moreover, this model can further branch to new knockout/in or transgenic mice that could shed light on human PsA disease development [63].

Another model used in the study of PsA, the porcine animal model, was chosen due to the anatomical and physiological similarities between porcine and human skin. In Gottingen minipigs, a model of transgenic animals was designed, expressing human $\beta 1$ or $\alpha 2$ integrin under the control of a promoter specific for suprabasal keratinocytes. In this model, biomarkers of skin homeostasis were found sustaining pro-inflammatory cytokine IL-1 $\alpha$ and an enhanced expression of the transcription factor c-Fos. These pro-inflammatory molecules are correlated with an activation of the MAPK pathway. This entire inflammatory milieu can favor the development of an inflammatory skin disease. As in the above mice models, in the pig models integrins' involvement in psoriasis was demonstrated as well. Increased expression of integrins $\alpha 2$ and/or $\beta 1$ induces enhanced keratinocytes proliferation and influx of T lymphocytes in the dermal and epidermal layers [64].

Animal models of PsA, although difficult in reproducing human disease are important for pre-clinical development of targeted drugs.

\section{Approved immune therapy for PsA}

There is a series of drugs approved for PsA, some of them approved by the US FDA, some by the European Medicines Agency (EMA) and some approved by both Agencies. FDA has approved the following drugs for PsA: adalimumab, aspirin, etanercept, methylprednisolone acetate, nonselective NSAIDs, prednisolone, triamcinolone acetonide, triamcinolone diacetate [65] while EMA approved leflunomide, etanercept, adalimumab, infliximab, and golimumab [66]. Recently reviewed, the therapeutic strategies for PsA can be classified in systemic agents (methotrexate, ciclosporin, leflunomide) and biologic agents such as TNF inhibitors (adalimumab, etanercept, infliximab, golimumab, certolizumab), anti IL-17 agents (ixekizumab, secukinumab), and anti IL-12p40 (ustekinumab) [67].

In the clinical management of PsA early treatment with anti-rheumatic drugs slows the progression of the disease, delivering a good life quality. Traditional methotrexate and cyclosporine are temporarily effective in PsA, but anti-TNF drugs, etanercept, inflix- imab and adalimumab are efficacious when patients are diagnosed with psoriasis and PsA. Recent clinical trials testing phosphodiesterase 4 inhibitors (apremilast), anti-IL-17 (secukinumab, brodalumab and ixekizumab) show encouraging results in psoriasis and/or PsA [68,69]. It was reported that around $30 \%$ of PsA patients are non-responders or resistant to drugs that block TNF- $\alpha$ [70]. Inhibition of T lymphocytes costimulatory pathway by using abatacept and alefacept and P40 fraction of IL-12 and IL-23 blockers (such as ustekinumab), has proven a good efficacy [71]. Apremilast, a phosphodiesterase, is a novel drug that lately opens other therapeutic possibilities as further elaborated. Inhibitors for CD20 and IL-1 can be future good therapeutical candidates [72,73]. As in RA, antiJAK drugs, targeting cytokine's signal transduction pathways, were shown to be effective in the treatment of psoriasis and probably a therapeutic extension to PsA is expected in the near future [74].

Leflunomide is an immunomodulatory drug that blocks the dihydroorotate dehydrogenase (DHODH) enzyme with consequent disruption of de novo pyrimidine biosynthesis, inhibiting thus $\mathrm{T}$ and $\mathrm{B}$ lymphocytes proliferation. Leflunomide was first approved by EMA for RA treatment and afterwards, the treatment indications extended to PsA, based on a multinational, randomized, double-blind study on active PsA. According to the Psoriatic Arthritis treatment Response Criteria (PsARC), this study found 59\% responders in the leflunomide group while only $29.7 \%$ in the placebo group [75]. Newly data from a prospective, multinational and observational study have shown more optimistic results, namely up to $86.4 \%$ of PsA patients achieved a PsARC response [76]. Only a few studies tested the efficacy of combined therapy in PsA, in contrast with RA, were studies abounded [77].

However, a recent retrospective study revealed a statistically significant improvement of the mean DAS28 and C-reactive protein at 12-16 weeks after the addition of leflunomide in the treatment of a small group of patients with PsA resistant to metotrexat monotherapy, these markers being the only soluble ones tested. Although leflunomide's anti-proliferative and anti-inflammatory effects were attributed to DHODH inhibition by its metabolite (A771726), additional mechanisms could be also involved [78].

Alefacept (a genetically engineered molecule designed to have a LFA-3 human antigen and immunoglobin G1 (human hinge $\mathrm{CH} 2-\mathrm{CH} 3 \gamma 1$-chain) dimer - LFA3Ig), an FDA approved drug for treatment of psoriasis was not approved for the European patients. When preparing the pre-clinical results for this drug, it was reported that the efficacy of the drug should be evaluated by genetic biomarkers, such as the 
modification in PBMC of tolerance-associated gene (TOAG-1) associated with proteomic biomarkers, for instance the expression of receptor for hyaluronic acid mediated migration (RHAMM) [79].

The results of an open-label extension study using alefacept and methotrexate (MTX) in both PsA and psoriasis patients have shown that PsA patients treated with MTX can have good clinical outcome after one or more courses of alefacept, without any additional toxicity [80].

Adalimumab, the first FDA-approved therapeutical monoclonal antibody, acts as a blocker of TNF- $\alpha$, preventing this cytokine to link to its specific receptor. This drug started as a RA drug and it was extended to PsA [81]. This year, investigating the effectiveness of the drug in comparison to other biologicals, it was concluded that adalimumab, etanercept and infliximab were the most effective drugs when assessing PsARC and Health Assessment Questionnaire (HAQ). Moreover, this study showed that assessing adalimumab, etanercept, golimumab and infliximab in PsA in terms of cost-effectiveness, etanercept is on the top of the four biologics evaluated [82].

Studying biomarkers that can be useful in monitoring tumor necrosis factor inhibitors (e.g., adalimumab) it was reported that a reduction in soluble MMP-3 and an increase in serum cartilage oligomeric matrix protein are independently associated with a good clinical response to this therapy applied in PsA [83].

Ustekinumab is a monoclonal antibody anti-IL-12 and -23 , cytokines known to mediate inflammatory processes. It can be used alone or with methotrexate; in 2009 it was approved for patients diagnosed with moderate-to-severe plaque psoriasis. This antibody received FDA approval in the end of 2013 for PsA treatment [84]. Results published this year regarding clinical efficacy have shown that ustekinumab prove beneficial improvements in PsA symptoms, including patients prior subjected to anti-TNF drugs [85].

Apremilast is an analog of thalidomide that inhibits phosphodiesterase IV (PDE4). This enzyme hydrolysis cyclic adenosine monophosphate (cAMP) to adenosine monophosphate, being the main enzyme involved in the cAMP homeostasis. cAMP induces a decrease in the TNF- $\alpha$ level, but the clinical tests are not as beneficial as one would might have expected. Five Phase III clinical trials are up to now in progress, measuring both inflammation status and radiographic evaluation [75]. The less beneficial effects of this inhibitor are due probably to the complex array of pathways cAMP is involved in, meaning that PDE 4 inhibition can directly lead to cAMP disturbances and/or cAMP signaling by itself produces other unknown effects [86].
Patients diagnosed with PsA, mainly those with moderate to severely active PsA should be treated with disease-modifying anti-rheumatic drugs (methotrexate), TNF inhibitors like adalimumab, etanercept, infliximab and golimumab or with their combination [87].

In the pursue of targeting immune proteins for new therapies in PsA, there are around 50 open clinical trials that focus mainly on TNF acting biologics [88].

Although there is an armentarium of immune biologics targeting PsA, the biomarkers that are studied and put in use in clinical trials for these therapies still lack both the number and the specificity.

\section{Conclusion \& future perspective}

The pathogenesis of PsA and the immunological background is still a subject of intense research, if not debate. If there is a genetic predisposition of developing this disease, mature immune cells and immune molecules (e.g., complement, cytokines) sustain the immune mechanisms that develop the disease, but the actual trigger that is 'conducting' these processes is still unknown [89]. The initiation trigger seems to be an unknown environmental factor, which underlies a chronic inflammation. The immune response generated upon initial trigger is thought to be defective, thus generating an auto-immune/auto-inflammatory condition. Cytokines that emerge due to the activation of lymphocytes with Th1 and Th17 functions (e.g TNF- $\alpha$ and IL-17) maintain this chronic inflammatory response acknowledged in the PsA patient's joints. Clinical trials using anti-IL-17A antibody therapy are still on-going, but studies regarding the interplay between the array of Th17-type cytokines (e.g., IL-17A, IL-17F, IL-22, IL-21) as regulatory pathways in PsA are still needed.

We believe that the pathogenesis complexity of PsA resides also in the psoriatic synovitis milieu, where intricate immune relations are emerging during disease development. Herein, immune cells collaborate directly and/or via secreted molecules, cells can change their phenotype, can repress or activate genes involved in the immune response during various stages of the disease. Innate immune response generates inflammatory cytokines driving effectors functions for immune and non-immune cells that sustain the chronic character of the synovitis. Ligands and receptors activated by transcription factors appending to regulatory loops trigger a range of complex signaling pathways and therefore, therapy must also seek a combination of drugs targeting multiple pathways. More so, drug development in PsA is challenging, because it has to restore the balance between innate and adaptive immunity, without hindering any other 'correct' immunological processes.

The heterogeneity and complexity of PsA pathogenesis requests complex methodological approaches. 
Going deeper in the pathogenesis of this disease, the associated genetic predisposition can lead to new immune biomarkers whether from the proteomic or genomic area and identify new therapeutic approaches.

As in any other pathologies, therapy that is triggered toward immunity arms can have deleterious effects, thus noninvasive diagnostic tools that can predict as early as possible the responders are of paramount importance and a closer collaboration between proteomics research and clinicians from the dermatology and rheumatology field would only have beneficial results in the management of PsA patients.

\section{Author contributions}

C Caruntu, D Boda, C Constantin and M Neagu have equally participated in designing, writing and revising the presented paper.

\section{Acknowledgements}

The authors would like to thank I Radu, certified translator in
Medicine-Pharmacy, certificate credentials: series E no. 0048, for professional linguistic assistance.

\section{Financial \& competing interests disclosure}

The study was supported from the following grants: POSDRU/159/1.5/G/141531, PN 09.33-01.01/2009, PN-II-ID-PCE2011-3-0918, PN-II-RU-TE-2011-3-0249. C Caruntu has a postdoctoral fellowship in the project POSDRU/159/1.5/G/141531. The authors have no other relevant affiliations or financial involvement with any organization or entity with a financial interest in or financial conflict with the subject matter or materials discussed in the manuscript apart from those disclosed.

No writing assistance was utilized in the production of this manuscript.

\section{Open access}

This work is licensed under the Creative Commons Attribution-NonCommercial 3.0 Unported License. To view a copy of this license, visit http://creativecommons.org/licenses/bync-nd/3.0/

\section{Executive summary}

- Psoriatic arthritis has a peculiar position in the Immunological Disease Continuum, being categorized as an auto-inflammatory disease comprising forms that can have or not MHC association. Thus, it has a distinctive feature from other rheumatic diseases in terms of its clinical, genetical, immunohistochemical and imaging characteristics.

- Psoriatic arthritis has a central immune backbone comprising inflammatory cascades and cytokine networks that are converging to a sustained inflammation that leads to altered tissues targeting two distinct organs (skin and joints).

- Due to its specific immune features, the immunopathophysiology, it seems intricately related to T lymphocytes that have specific clones for both skin and synovium. Abnormal innate and adaptive immune activation to an external trigger can lead to the chronic disease stage if there is also a genetic predisposition (e.g., HLA-C 'PsA gene', with HLA-CW*0602 as risk allele).

- Proteomics comes to rescue for the psoriatic arthritis ambiguous biomarker field. Multiplex-biomarker detection could differentiate between rheumatoid arthritis and psoriatic arthritis and a soluble biomarkers profile can furbish an important tool for early stratification of patients on the bridge of developing the arthritic form and thus identifying a worse clinical outcome. There is a need of developing studies that use proteomic technologies in order to enlarge the panel of discovered biomarkers.

- Therapy in psoriatic arthritis has an armentarium of systemic and biologic agents, such as TNF inhibitors, anti IL-17 agents, anti IL-12p40, but on-going clinical trials testing others, like phosphodiesterase 4 inhibitors show future encouraging results.

\section{References}

Papers of special note have been highlighted as:

- of interest; $\bullet$ of particular interest

1 Lloyd P, Ryan C, Menter A. Psoriatic arthritis: an update. Arthritis 176298 (2012).

2 Gladman DD, Antoni C, Mease P et al. Psoriatic arthritis: epidemiology, clinical features, course, and outcome. Ann. Rheum. Dis. 64(Suppl. 2), ii14-ii17 (2005).

3 Chandran V. Soluble biomarkers may differentiate psoriasis from psoriatic arthritis. J. Rheumatol. 39(89), 65-66 (2012).
4 Paukkonen K, Naukkarinen A, Horsmanheimo M. The development of manifest psoriatic lesions is linked with the invasion of CD81 T cells and CD11c1 macrophages into the epidermis. Arch. Dermatol. Res. 284, 375-379 (1992).

5 Villadsen LS, Schuurman J, Beurskens F et al. Resolution of psoriasis upon blockade of IL-15 biological activity in a xenograft mouse model. J. Clin. Invest. 112, 1571-1580 (2003).

6 Antoni CE, Kavanaugh A, van der Heijde D et al. Two-year efficacy and safety of infliximab treatment in patients with active psoriatic arthritis: findings of the Infliximab Multinational Psoriatic Arthritis 
Controlled Trial (IMPACT). J. Rheumatol. 35(5), 869-876 (2008).

7 Härle P. Detecting psoriasis arthritis early in the disease course - why this is important and how dermatologists and rheumatologists can successfully cooperate? In: Psoriasis, Soung J (Ed.) 65-78 (2012).

8 Park H, Bourla AB, Kastner DL et al. Lighting the fires within: the cell biology of autoinflammatory diseases. Nat. Rev. Immunol. 12, 570-580 (2012).

9 McGonagle D. Enthesitis: an autoinflammatory lesion linking nail and joint involvement in psoriatic disease. J. Eur. Acad. Dermatol. Venereol. 23(Suppl. 1), 9-13 (2009).

10 Schön MP, Boehncke WH. Psoriasis. NEJM 352(18), 1899-1912 (2005).

11 Caruntu C, Boda D. Evaluation through in vivo reflectance confocal microscopy of the cutaneous neurogenic inflammatory reaction induced by capsaicin in human subjects. J. Biomed. Opt. 17(8), 085003 (2012).

12 Wolberink EA, van Erp PE, Teussink MM et al. Cellular features of psoriatic skin: imaging and quantification using in vivo reflectance confocal microscopy. Cytom. Part B: Clin. Cytom. 80(3), 141-149 (2011).

13 Takahashi $\mathrm{H}$, Tsuji $\mathrm{H}$, Hashimoto $\mathrm{Y}$ et al. Cell proliferation and cytokine induction by TNF- $\alpha$ of psoriatic keratinocytes are not different from normal keratinocytes in vitro. Indian J. Dermatol. 54(3), 237-239 (2009).

14 Boda D, Diaconeasa A, Neagu M et al. Studiu privind eficacitatea terapiei fotodinamice $\mathrm{Cu}$ acid 5-aminolevulinic in psoriazis vulgar. Dermatovenerol. (Buc.) 53, 85-90 (2008).

15 Aydin SZ, Ash ZR, Tinazzi I et al. The link between enthesitis and arthritis in psoriatic arthritis: a switch to a vascular phenotype at insertions may play a role in arthritis development. Ann. Rheum. Dis. 72(6), 992-995 (2013).

16 Gladman DD Helliwell PS, Khraishi M et al. Dermatology screening tools: project update from the GRAPPA 2012 annual meeting. J. Rheumatol. 40 (8), 1425-1427 (2013).

17 Grazio S, Razdorov G, Erjavec I et al. Differential expression of proteins with heparin affinity in patients with rheumatoid and psoriatic arthritis: a preliminary study. Clin. Exp. Rheumatol. 31(5), 665-671 (2013).

18 Chandra PE, Sokolove J, Hipp BG et al. Novel multiplex technology for diagnostic characterization of rheumatoid arthritis. Arthritis Res. Ther 13(3), R102 (2011).

-• Highly cited paper that describes a novel automated multiplex biomarker assay that could differentiate between rheumatoid arthritis and psoriatic arthritis.

19 Dalmady S, Kiss M, Kepiro L et al. Higher levels of autoantibodies targeting mutated citrullinated vimentin in patients with psoriatic arthritis than in patients with psoriasis vulgaris. Clin. Dev. Immunol. 474028 (2013).

20 Ramonda R, Modesti V, Ortolan A et al. Serological markers in psoriatic arthritis: promising tools. Exp. Biol. Med. (Maywood). 238(12), 1431-1436 (2013).

-• An original paper that identifies specific biomarkers useful for the early detection of psoriatic arthritis.
21 Schlaak JF, Buslau M, Jochum W et al. T cells involved in psoriasis vulgaris belong to the Th1 subset. J. Invest. Dermatol. 102, 145-149 (1994).

22 Ong PY, Hamid QA, Travers JB et al. Decreased IL-15 may contribute to elevated $\operatorname{IgE}$ and acute inflammation in atopic dermatitis. J. Immunol. 168, 505-510 (2002).

23 Pattison E, Harrison BJ, Griffiths CE et al. Environmental risk factors for the development of psoriatic arthritis: results from a case-control study. Ann. Rheum. Dis. 67(5), 672-676 (2008).

- Highly cited report that focuses on potential environmental risk factors associated with the onset of psoriatic arthritis.

24 Liang SC, Tan XY, Luxenberg DP et al. Interleukin (IL)-22 and IL-17 are coexpressed by Th17 cells and cooperatively enhance expression of antimicrobial peptides. J. Exp. Med. 203(10), 2271-2279 (2006).

25 Lories RJ, de Vlam K. Is psoriatic arthritis a result of abnormalities in acquired or innate immunity? Curr. Rheumatol. Rep. 14(4), 375-382 (2012).

26 Cañete JD. Biopathology of the synovial membrane in psoriatic arthritis. Reumatol.Clin. 8(1), S10-S4 (2012).

27 Wenink MH, Santegoets KC, Butcher J et al. Impaired dendritic cell proinflammatory cytokine production in psoriatic arthritis. Arthritis Rheum. 63(11), 3313-3322 (2011).

28 Yoo IS, Lee JH, Song ST et al. T-helper 17 cells: the driving force of psoriasis and psoriatic arthritis. Int. J. Rheum. Dis. 15(6), 531-537 (2012).

29 Pène J, Chevalier S, Preisser L et al. Chronically inflamed human tissues are infiltrated by highly differentiated Th17 lymphocytes. J. Immunol. 180(11), 7423-7430 (2008).

30 Aochi S, Tsuji K, Sakaguchi M et al. Markedly elevated serum levels of calcium-binding S100A8/A9 proteins in psoriatic arthritis are due to activated monocytes/ macrophages. J. Am. Acad. Dermatol. 64(5), 879-887 (2011).

31 Ballanti E, Perricone C, di Muzio G et al. Role of the complement system in rheumatoid arthritis and psoriatic arthritis: relationship with anti-TNF inhibitors. Autoimmun. Rev. 10(10), 617-623 (2011).

32 Bonecchi R, Borroni EM, Anselmo A et al. Regulation of D6 chemokine scavenging activity by ligand- and Rab11dependent surface up-regulation. Blood 112(3), 493-503 (2008).

33 Singh MD, King V, Baldwin $\mathrm{H}$ et al. Elevated expression of the chemokine-scavenging receptor D6 is associated with impaired lesion development in psoriasis. Am. J. Pathol. 181(4), 1158-1164 (2012).

34 Flytlie HA, Hvid M, Lindgreen E et al. Expression of MDC/ CCL22 and its receptor CCR 4 in rheumatoid arthritis, psoriatic arthritis and osteoarthritis. Cytokine 49(1), 24-29 (2010).

35 Martin MP, Nelson G, Lee JH et al. Cutting edge: susceptibility to psoriatic arthritis: influence of activating killer Ig-like receptor genes in the absence of specific HLA-C alleles. J. Immunol. 169(6), 2818-2822 (2002). 
36 Antonelli A, Fallahi P, Delle Sedie A et al. High values of Th1 (CXCL10) and Th2 (CCL2) chemokines in patients with psoriatic arthtritis. Clin. Exp. Rheumatol. 27(1), 22-27 (2009).

37 Antonelli A, Ferrari SM, Giuggioli D et al. Chemokine (C-X-C motif) ligand (CXCL) 10 in autoimmune diseases. Autoimmun. Rev. 13(3), 272-280 (2014).

Zhang AL, Colmenero P, Purath U et al. Natural killer cells trigger differentiation of monocytes into dendritic cells. Blood 110(7), 2484-2493 (2007).

39 Jongbloed SL, Lebre MC, Fraser AR et al. Enumeration and phenotypical analysis of distinct dendritic cell subsets in psoriatic arthritis and rheumatoid arthritis. Arthritis Res. Ther. 8(1), R15 (2006).

40 Candia L, Marquez J, Hernandez C et al. Toll-like receptor-2 expression is upregulated in antigen-presenting cells from patients with psoriatic arthritis: a pathogenic role for innate immunity? J. Rheumatol. 34(2), 374-379 (2007).

41 Daoussis D, Antonopoulos I, Andonopoulos AP, Liossis $\mathrm{SN}$. Increased expression of CD154 (CD40L) on stimulated T-cells from patients with psoriatic arthritis. Rheumatology (Oxford) 46(2), 227-231 (2007).

42 Myśliwiec H, Flisiak I, Baran A et al. Evaluation of CD40, its ligand $\mathrm{CD} 40 \mathrm{~L}$ and $\mathrm{Bcl}-2$ in psoriatic patients. Folia Histochem. Cytobiol. 50(1), 75-79 (2012).

43 Liu Y, Helms C, Liao W et al. A genome-wide association study of psoriasis and psoriatic arthritis identifies new disease loci. PLoS Genetics 4(3), e1000041 (2008).

44 Popa OM, Kriegova E, Popa L. Association study in Romanians confirms IL23A gene haplotype block rs2066808/rs11171806 as conferring risk to psoriatic arthritis. Cytokine 63(1), 67-73 (2013).

45 McGonagle D, Palmou Fontana N, Tan AL, Benjamin M. Nailing down the genetic and immunological basis for psoriatic disease. Dermatology 221(1), 15-22 (2010).

46 Rahman P, Elder JT. Genetics of psoriasis and psoriatic arthritis: a report from the GRAPPA 2010 annual meeting. J. Rheumatol. 39(2), 431-433 (2012).

47 Eder L, Chandran V, Pellett F et al. Differential human leucocyte allele association between psoriasis and psoriatic arthritis: a family-based association study. Ann. Rheum. Dis. 71(8), 1361-1365 (2012).

48 Chandran V, Bull SB, Pellett FJ et al. Killer-cell immunoglobulin-like receptor gene polymorphisms and susceptibility to psoriatic arthritis. Rheumatology (Oxford) 53(2), 233-239 (2014).

49 Bowes J, Ho P, Flynn E et al. Comprehensive assessment of rheumatoid arthritis susceptibility loci in a large psoriatic arthritis cohort. Ann. Rheum. Dis. 71(8), 1350-1354 (2012). Chandran V. The genetics of psoriasis and psoriatic arthritis. Clin. Rev. Allergy. Immunol. 44(2), 149-156 (2013).

51 Tilleman K, Deforce D. Proteomics in rheumatology. Expert Rev. Proteomics 5(6), 755-759 (2008).

52 de Seny D, Fillet M, Ribbens C et al. Monomeric calgranulins measured by SELDI-TOF mass spectrometry and calprotectin measured by ELISA as biomarkers in arthritis. Clin. Chem. 54, 1066-1075 (2008).
53 Chandran V, Cook RJ, Edwin J et al. Soluble biomarkers differentiate patients with psoriatic arthritis from those with psoriasis without arthritis. Rheumatology 49, 1399-1405 (2010).

- Important report that identifies soluble biomarkers that distinguish patients diagnosed with psoriatic arthritis from those diagnosed with psoriasis.

54 Ryu J, Park SG, Park BC et al. Proteomic analysis of psoriatic skin tissue for identification of differentially expressed proteins: up-regulation of GSTP1, SFN and PRDX2 in psoriatic skin. Int. J. Mol. Cell. Med. 28(5), 785-792 (2011).

55 Bostan M, Brasoveanu LI, Livescu A et al. Effects of synovial fluid on the respiratory burst of granulocytes in rheumatoid arthritis. J. Cell. Mol. Med., 5(2), 188-194 (2001).

56 Piruzian E, Bruskin S, Ishkin A et al. Integrated network analysis of transcriptomic and proteomic data in psoriasis. BMC Syst. Biol. 4, 41-50 (2010).

57 Haddad A, Chandran V. Arthritis mutilans. Curr. Rheumatol. Rep. 15(4), 321 (2013).

58 Nickoloff BJ, Bonish B, Huang BB, Porcelli SA. Characterization of a $\mathrm{T}$ cell line bearing natural killer receptors and capable of creating psoriasis in a SCID mouse model system. J. Dermatol. Sci. 24, 212-225 (2000).

59 Tang F, Sally B, Ciszewski C et al. Interleukin 15 primes natural killer cells to kill via NKG2D and cPLA2 and this pathway is active in psoriatic arthritis. PLoS ONE 8(9), e76292 (2013).

60 Conrad C, Boyman O, Tonel G et al. Alpha1beta1 integrin is crucial for accumulation of epidermal $\mathrm{T}$ cells and the development of psoriasis. Nat. Med. 13(7), 836-842 (2007).

61 Sano S, Chan KS, Carbajal S et al. Stat3 links activated keratinocytes and immunocytes required for development of psoriasis in a novel transgenic mouse model. Nat. Med. 11, 43-49 (2005).

62 Wang H, Peters T, Sindrilaru A et al. TGF- $\beta$-dependent suppressive function of Tregs requires wild-type levels of CD18 in a mouse model of psoriasis. J. Clin. Invest. 118, 2629-2639 (2008)

63 Retser E, Schied T, Skryabin BV et al. Doxycycline-induced expression of transgenic human tumor necrosis factor $\alpha$ in adult mice results in psoriasis-like arthritis. Arthritis Rheum. 65(9), 2290-2300 (2013).

64 Staunstrup NH, Madsen J, Primo MN et al. Development of transgenic cloned pig models of skin inflammation by DNA transposon-directed ectopic expression of human beta1 and alfa2 Integrin. PLoS ONE 7(5), e36658 (2012).

65 US FDA. Rheumathology Therapeutics: Drugs and Biologics.

www.fda.gov

66 European Medicine Agency - European public assessment reports (EPAR) for human medicines (keyword search for 'Psoriatic arthritis'.

www.ema.europa.eu

67 Gan EY, Chong WS, Tey HL. Therapeutic strategies in psoriasis patients with psoriatic arthritis: focus on new agents. BioDrugs 27(4), 359-373 (2013). 
68 Schett G, Wollenhaupt J, Papp K et al. Oral apremilast in the treatment of active psoriatic arthritis: results of a multicenter, randomized, double-blind, placebo-controlled study. Arthritis Rheumatol. 64(10), 3156-3167 (2012).

- Multicentric evaluation of the efficacy and safety of apremilast, a phosphodiesterase 4 inhibitor, in the treatment of active psoriatic arthritis.

69 Mease PJ, Genovese MC, Greenwald MW et al. Brodalumab, an anti-IL17RA monoclonal antibody, in psoriatic arthritis. NEJM. 370 (24), 2295-2306 (2014).

70 Coates LC, Cawkwell LS, Ng NW et al. Sustained response to long-term biologics and switching in psoriatic arthritis: results from real life experience. Ann. Rheum. Dis. 67(5), 717-7179 (2008).

71 Kavanaugh A, Ritchlin C, Rahman P et al. PSUMMIT-1 and 2 Study Groups. Ustekinumab, an anti-IL-12/23 p40 monoclonal antibody, inhibits radiographic progression in patients with active psoriatic arthritis: results of an integrated analysis of radiographic data from the Phase 3, multicentre, randomised, double-blind, placebo-controlled PSUMMIT-1 and PSUMMIT-2 trials. Ann. Rheum. Dis. 73(6), 10001006 (2014).

72 Jimenez-Boj E, Stamm TA, Sadlonova M et al. Rituximab in psoriatic arthritis: an exploratory evaluation. Ann. Rheum. Dis. 71(11), 1868-1871 (2012).

73 Jung N, Hellmann M, Hoheisel R et al. An open-label pilot study of the efficacy and safety of anakinra in patients with psoriatic arthritis refractory to or intolerant of methotrexate (MTX). Clin. Rheumatol. 29(10), 1169-1173 (2010).

74 Papp KA, Menter A, Strober B et al. Efficacy and safety of tofacitinib, an oral Janus kinase inhibitor, in the treatment of psoriasis: a Phase $2 \mathrm{~b}$ randomized placebo-controlled doseranging study. Br. J. Dermatol. 167(3), 668-677 (2012).

75 European Medicines Agency: Leflunomide: European Public Assessment Reports (EPAR) - Scientific Discussion. www.ema.europa.eu

76 Behrens F, Finkenwirth C, Pavelka K et al. Leflunomide in psoriatic arthritis: results from a large European prospective observational study. Arthritis Care Res. (Hoboken) 65(3), 464-470 (2013).

77 Bird P, Griffiths H, Tymms K et al. The SMILE study safety of methotrexate in combination with leflunomide in rheumatoid arthritis. J. Rheumatol. 40(3), 228-235 (2013).

78 Sakellariou GT, Sayegh FE, Anastasilakis AD, Kapetanos GA. Leflunomide addition in patients with articular manifestations of psoriatic arthritis resistant to methotrexate. Rheumatol. Int. 33(11), 2917-2920 (2013).

79 Keeren K, Friedrich M, Gebuhr I et al. Expression of tolerance associated gene-1, a mitochondrial protein inhibiting $\mathrm{T}$ cell activation, can be used to predict response to immune modulating therapies. J. Immunol. 183, 4077-4087 (2009).

80 Mease PJ, Reich K. Alefacept in Psoriatic Arthritis Study Group Alefacept with methotrexate for treatment of psoriatic arthritis: open-label extension of a randomized, double-blind, placebo-controlled study. J. Am. Acad. Dermatol. 60 (3), 402-411 (2009).

81 Menter A, Tyring SK, Gordon K et al. Adalimumab therapy for moderate to severe psoriasis: a randomized, controlled Phase III trial. J. Am. Acad. Dermatol. 58(1), 106-115 (2008).

82 Cawson MR, Mitchell SA, Knight C et al. Systematic review, network meta-analysis and economic evaluation of biological therapy for the management of active psoriatic arthritis. BMC Musculoskelet. Dis. 15(1), 26-36 (2014).

83 Chandran V, Shen H, Pollock RA et al. Soluble biomarkers associated with response to treatment with tumor necrosis factor inhibitors in psoriatic arthritis. J. Rheumatol. 40(6), 866-871 (2013).

84 STELARA $^{\circledR}$. www.STELARAinfo.com

85 Ritchlin C, Rahman P, Kavanaugh A et al. Efficacy and safety of the anti-IL-12/23 p 40 monoclonal antibody, ustekinumab, in patients with active psoriatic arthritis despite conventional non-biological and biological antitumour necrosis factor therapy: 6-month and 1-year results of the Phase 3, multicentre, double-blind, placebo-controlled, randomised PSUMMIT 2 trial. Ann. Rheum. Dis. 73(6), 990-999 (2014).

86 Conti M, Richter W, Mehats C et al. Cyclic AMP-specific PDE4 phosphodiesterases as critical components of cyclic AMP signaling. J. Biol Chem. 278, 5493-5496 (2003).

87 ClinicalTrials.gov. http://clinicaltrials.gov

88 Papoutsaki M, Costanzo A. Treatment of psoriasis and psoriatic arthritis. BioDrugs 27(1), 3-12 (2013).

89 Chimenti MS, Ballanti E, Perricone C et al. Immunomodulation in psoriatic arthritis: focus on cellular and molecular pathways. Autoimmun. Rev. 12(5), 599-606 (2013). 